\title{
WEB BASED 3D VISUALISATION OF TIME-VARYING AIR QUALITY INFORMATION
}

\author{
U. Isikdag ${ }^{1 *}$, K. Sahin ${ }^{1}$ \\ 1. MSGSU, Dept. of Informatics, Mimar Sinan Fine Art University, Istanbul,Turkey (umit.isikdag,kemal.sahin)@ msgsu.edu.tr
}

Commission IV, WG IV/10

KEY WORDS: 3D, Visualisation, Air Quality, Cesium, PostGIS, Time

\begin{abstract}
:
Many countries where the industrial development and production rates are high face many side effects of low air quality and air pollution. There is an evident correlation between the topographic and climatic properties of a location and the air pollution and air quality on that location. As the variation of air quality is dependent on location, air quality information should be acquired, utilised, stored and presented in form of Geo-Information. On the other hand, as this information is related with the health concerns of public, the information should be available publicly, and needs to be presented through an easily accessible medium and through a commonly used interface. Efficient storage of time-varying air quality information when combined with an efficient mechanism of $3 \mathrm{D}$ web-based visualisation would help very much in dissemination of air quality information to public. This research is focused on web-based 3D visualisation of time-varying air quality data. A web based interactive system is developed to visualise pollutant levels that were acquired as hourly intervals from more than 100 stations in Turkey between years 2008 and 2017. The research also concentrated on visualisation of geospatial high volume data. In the system, visualisation can be achieved on-demand by querying an air pollutant information database of 10.330 .629 records and a city object database with more than 700.000 records. The paper elaborates on the details of this research. Following a background on air quality, air quality models, and Geo-Information visualisation, the system architecture and functionality is presented. The paper concludes with results of usability tests of the system.
\end{abstract}

\section{INTRODUCTION}

Air quality has an important influence on of the health and quality of life. In many of the developed countries majority of the population lives in the urban and suburban areas, where the impact of air quality to everyday activities is major. Many countries such as China where the industrial development and production rates are high, face many side effects of low air quality and air pollution. It is clearly identified in research that air pollution, which first appeared in the 19th century with industrial revolution and the side effects of the pollution, is closely affecting many life zones. (Boubel, 1994). Historical records indicate that on Friday 5th December 1952, a thick yellow smog brought the British capital to a standstill for four days and is estimated to have killed more than 4,000 people in London. The first series of serious incidents related to air pollution in Turkey, were experienced in Ankara, in 1970s. Inhabitants of Ankara have spent days in hospitals regarding respiratory problems. Today, low air quality and air pollution has become a widespread problem globally, which shows significant increases not only in the big cities but also in the rural areas. The research points out that the air quality decreases directly proportional to the population growth and industrial development level. Another factor that has a direct impact on low air quality is our transportation systems. Both industrial production and transportation sectors heavily rely on fossil fuels. The widespread increase in fossil fuel consumption, such as coal, lignite and oil, directly increases the air pollution and the suspension duration of the all particles and cancerous pollutants in the air. There is an evident correlation between the topographic and climatic properties of a location and the air pollution and air quality on that location. For that reason, topographic and meteorological parameters of the region are investigated in order to determine the size and proportion of air pollution (Jacob, 1999). Thus, it can be clearly stated that variation of air quality is dependent on location, and in other words, air quality information should be acquired, utilised, stored and presented in form of Geo-Information. On the other hand as this information is related with the health concerns of the public, the information should be available publicly, and needs to be presented through an easily accessible medium and also through a highly usable user interface. The recent developments in spatial databases and visualisation tools provide new opportunities in efficient storage of air quality information, which is usually stored in a time-varying nature (i.e. in form of many readings taken from different stations during time period). New opportunities related to visualisation of information also emerge through virtual globes where information can be visualised in 3D using regular web browsers. Efficient storage of time-varying air quality information when combined with an efficient mechanism of 3D web based visualisation would provide an easy interface for users and would help very much in dissemination of air quality information to public.

The prototype developed in this research visualises the pollutant levels that were acquired as hourly intervals from more than 100 stations in Turkey between years 2008 and 2017. The two attributes of the air quality information in this case, i.e. being high-volume and time varying along with its spatial nature, makes the visualisation of it a challenging research problem. The novelty of the system is in acting as a proof-of-concept for demonstrating various approaches for web based 3D visualisation of high volume and time-varying air quality information. In this context, this research aimed to develop a web based prototype system for 3D Visualisation of Time Varying Air Quality Information. The objectives of the research were, to collect time varying air quality data along with its spatial dimension, to define and implement a spatial data storage mechanism, to design and implement the visualization system and finally to test the usability of the system. This paper elaborates on the details of this research. The paper also includes the results of usability test for the system. 


\section{BACKGROUND}

\subsection{Metrics and Measures on Air Quality}

Air pollution is defined as the existence of intensive amount of substances and particles that damage the ecological balance in the atmosphere. Increase of air pollution adversely affect the air quality. A special index for air quality has been developed to accurately identify and track factors that create adverse effects and affect human health and living (Spellman, 2009). Air Quality Index (AQI) is known as the index used to report air quality on a daily basis. It contains information on how clean or dirty the air of the area is, and what kind of health effects can occur. Air quality index is a simple way to report daily air quality. The index can provide limit values for different pollutants and particulate matter. In general practice, air quality index is calculated for 5 basic pollutants. These are particulate matter-PM, carbon monoxide-CO, sulphur dioxide-SO2 and nitrogen dioxide-NO2, ozone-O3. Air quality index limits have been determined for each of these pollutants (WHO, 2000). The pollutant group consisting of solid particles and liquid droplets, which are so small as to be suspended in the air, is called the particulate matter (Ahrens, 2000). The aerodynamic diameters of the particulate materials can vary from 0.1 micrometres to 30 micrometres. Particles smaller than 10 micrometres in diameter are called PM10 and are important for health effects. (Polichetti et al., 2009; Spellman, 2009). Throat pain and cough may be acute effects because of the exposure to PM10, chronic diseases such as bronchitis, chronic obstructive pulmonary disease (COPD) and asthma have also been reported as the adverse effects of PM10 exposure (MacNee and Donaldson, 1999). In last 30 years, many countries in Asia faced problems caused by low air quality and have taken preventive measures. For instance, in Turkey, Turkish Air Quality Protection Regulation (MOE, 1986) had foreseen the SO2 and PM10 short-term limits of $400 \mu \mathrm{g} / \mathrm{m} 3$ and $300 \mu \mathrm{g} / \mathrm{m} 3$ as well as long-term limit values of $150 \mu \mathrm{g} / \mathrm{m} 3$ for both. In 1986 National legislation also regulated the 8-month winter period urban air quality as 250 $\mu \mathrm{g} / \mathrm{m} 3$ for $\mathrm{SO} 2$ and $200 \mu \mathrm{g} / \mathrm{m} 3$ for PM10 (MOE, 1986). In 1996, World Health Organization and European Commission regulations introduced new air quality limits (Leeuwen, 1997). The Ministry of Environment and Urban Planning Issued Air Quality Assessment and Management Circular and the Regulation on Air Quality Assessment and Management (AQEMR) to reduce PM10 levels. The plan was to reduce allowable limit values gradually and to reach the European Union's limit values in 2019. Today, the Turkish Authorities tend to make regulations to make air pollutions to stay under EU and WHO limits. Turkish Air Quality Index is adopted from US EPA index and considers the EPA limits. These limits for each pollutant will be provided in the Visualisation of Information section of the paper.

\subsection{Visualisation of 3D and Time-Varying Geo-Information}

The spatio-temporal visualization, which is created by spatiotemporal information mapping, is an information visualization technique useful for specifying the feature area from simulation and measurement results. (Miyamura, 2010). This technique provides guidelines for selection of proper exploratory techniques depending on the characteristics of data to analyze and the goals of analysis (Andrienko et al, 2003).Visualisation of Geo-Information in two and three dimensions has been the core component of standalone or web based Geographical Information Systems. Visualisation of 3D Geo-Information/3D
City Models/3D Buildings has been a very active research topic in last 10 years. Some recent studies in the field include, Gesquière and Manin (2012) who presented an architecture for 3D visualisation of CityGML objects by using the WebGL modelling language. Jochem and Goetz (2012) presented a method to transform the crowdsourced OpenStreetMap data into a 3D city model. Contrary to other existing approaches towards OpenStreetMap 3D, the authors combined emerging and established Web technologies such as XML3D, allowing an easy consumption in arbitrary web browsers. Li et al (2012) provided an architecture for real time visualisation of geospatial big data. The authors used a multi-level cache approach to improve data loading efficiency. The unified management mechanism of caches is accomplished on three levels, including: the client memory cache (CMC) oriented to sharing application, the client file cache (CFC) organized by index, as well as the application server memory cache (ASMC) of structural consistency. Auer (2012) explained the use JavaScript 3D graphics API (and WebGL) to perform client-side parallel real-time computations of $2 \mathrm{D}$ or $2.5 \mathrm{D}$ spatial raster algorithms on the graphics card. Visualisations can be done for various purposes ranging from noise pollution mapping to energy demand. For example, Kaden and Kolbe (2014) presented a research focused on the city-wide estimation of the energy demands of buildings including heating, electricity and warm water energy in the city of Berlin using available official geobase and statistical data integrated within the Energy Atlas Berlin. The authors provided a visualisation using 3D buildings to show the calculated energy demand of each building. Zardiny and Hakimpour (2015) indicated that 3D Spatial Web Services allow on the fly construction and on demand presentation of the 3D cadastral models via Web.3D representations of city objects, which can be populated from $2 \mathrm{D}$, or $3 \mathrm{D}$ vector models. 3D representations of city objects can also be generated (reconstructed) using images. Among many studies, i.e. Homainejad (2015) provides an approach for object detection extraction from images to generate $3 \mathrm{D}$ building representations. A similar approach, which uses oblique images for reconstruction of building models, is explained in Ozerbil et al. (2015). Their study also involved automatic texturing of the reconstructed 3D Building Models. Another commonly used technique in 3D City Model generation is procedural modelling. For example, Tsiliakou et al (2015) explained that this algorithmic modelling concept is based on the principle that all real-world buildings are defined by rules, where repetitive patterns and hierarchical components describe their geometry. The detailed geometries of the model are derived from the application of CGA (Computer Generated Architecture) shape grammars on selected footprints, and the process results in a final 3D model, optimally describing the built environment. Klimke et al (2014) provided a a new approach for provisioning of massive, virtual 3D city models on different platforms such as web browsers, smartphones or tablets, by means of an interactive map assembled from artificial oblique image tiles. The key concept was to synthesize such images of a virtual 3D city model by a $3 \mathrm{D}$ rendering service in a preprocessing step. This service encapsulated model handling and 3D rendering techniques for high quality visualization of massive 3D models. By generating image tiles using this service, the 3D rendering process is shifted from the client side, which provides major advantages: (a) the complexity of the 3D city model data is decoupled from data transfer complexity (b) the implementation of client applications is simplified significantly as 3D rendering is encapsulated on server side (c) 3D city models can be easily deployed for and used by a large number of concurrent users, leading to a high degree of scalability of the overall approach 
The recent studies on visualisation of 3D Geo-Information involved the use of Virtual Globes. For example, Christen et al. (2012) explained The OpenWebGlobe project. The OpenWebGlobe SDK consisted of two main parts: i.) the OpenWebGlobe Viewer part a JavaScript Library which allows the integration of the OpenWebGlobe into custom webapplications, ii.)the OpenWebGlobe Processing Tools a bundle of tools for bulk data processing, e.g., tiling or resampling of large geospatial data sets. Brovelli et al (2014) stated that the dawn of GeoWeb 2.0, the geographic extension of Web 2.0, has opened new possibilities in terms of online dissemination and sharing of geospatial contents, thus laying the foundations for a fruitful development of Volunteered Geographic Information (VGI) systems. The authors conducted a study to investigate the extension of VGI applications, which are quite mature in the traditional bi-dimensional framework, up to the third dimension by means of virtual globes. The participative application (social virtual globe), which is built using the open source NASA World Wind virtual globe, is focused on the cultural and tourism heritage of Como city, located in Northern Italy. Using the tool users can create and manage customized projects and populate a catalogue of cartographic layers, which is available to the entire community. Together with historical maps and the current cartography of the city, the system is also able to manage geo-tagged data, which come from user field-surveys performed through mobile devices to report POIs (Points of Interest). As information on air quality consists of measurement results taken at specific times for a certain period the nature of this information is time-varying. Visualisation of Time-Varying 3D Air Quality Information is presented in Lu et al (2009). The authors stated that It is often challenging to handle 3D air quality datasets due to their large number of time steps and multiple interactional chemicals. In order to overcome this barrier, they designed and generated knowledge templates for visually analysing multi-field, time-varying $3 \mathrm{D}$ air quality data. Web Based 3D visualisation of Environmental Information is discussed in Herman and Reznik (2015). The authors presented a proof-of-concept application where the developed 3D visualization shows a terrain model, 3D buildings, noise pollution, and other related information. There were also studies on visualisation of air quality information together with City Models using the virtual globes. For example, San Jose (2012) visualized air quality index values on Google Earth, a threedimensional virtual globe also utilizing CityGML. A similar study was conducted on Zhu et al. (2016) again on a virtual globe platform CesiumJS, and utilizing CityGML. In fact, although these provide visualisation of air quality information in $3 \mathrm{D}$, none of those 2 studies were dealing with big geospatial data visualisation, which was one of the main focuses of the study explained in this paper.

\section{THE PROTOTYPE DEVELOPMENT AND IMPLEMENTATION}

Along with the aim of 3D Web Based Visualisation of Time Varying High Volume Air Quality Information, the system is developed in 3 stages. The system is developed as a "proof-ofconcept prototype" for establishing a web based 3D visualisation of high-volume time-varying air quality information. Thus, as this is a prototyping effort, the design requirements of the system are determined by the researchers only. Further user requirement analysis needs to be done to determine real-life design requirements if the system would be developed and implemented for a real life scenario. A use case diagram that is developed based on the input from the researchers is provided in Figure 1.

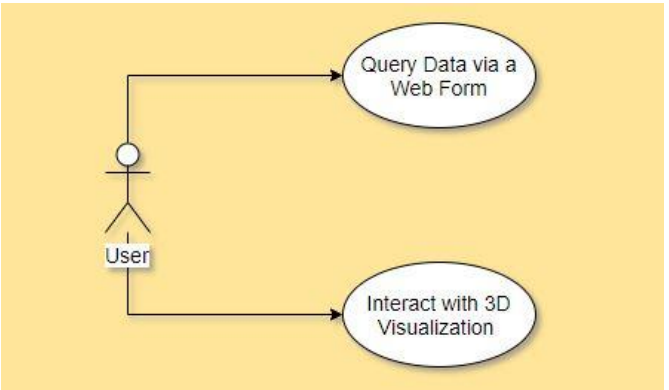

Figure 1: Use Cases of the Prototype

First stage of the effort involved the acquisition of air quality information from National Air Quality Monitoring Web Application, second stage of the effort was development and implementation of the system. The third stage involved a test to evaluate the usability of the system. This section explains first two stages, while the results of the testing stage is summarized in the conclusion section.

\subsection{Data Acquisition}

The procedures and principles regarding the management and monitoring of air quality in Turkey have been determined by the "Air Quality Assessment and Management Regulation (AQAAM)" which is fully compatible with the European Union (EU) environmental legislation. This regulation is set for Turkey with the aim of reaching the air quality levels of the EU countries. With this regulation, basically 13 pollutants (SO2, PM10, NOx, ..) are monitored. Following the establishment of the (AQAAM) regulation a National Air Quality Monitoring Network that was established throughout Turkey between 2005 2007. The network contains $100+$ monitoring stations and mobile monitoring vehicles, which store 13 different parameters for air quality measurement in Turkey, in form of hourly measurement results. The data is stored by Ministry of Environment and Urbanization and disseminated through an internally developed web application [http://www.havaizleme.gov.tr]. The National Air Quality Monitoring Web Application presents most recent measurement results via a $2 \mathrm{D}$ web map, and historical information is available for download on demand in form of CSV files. An overall architecture of the Ministry of Environment and Urbanization's Air Quality Monitoring System is illustrated in Figure 2.

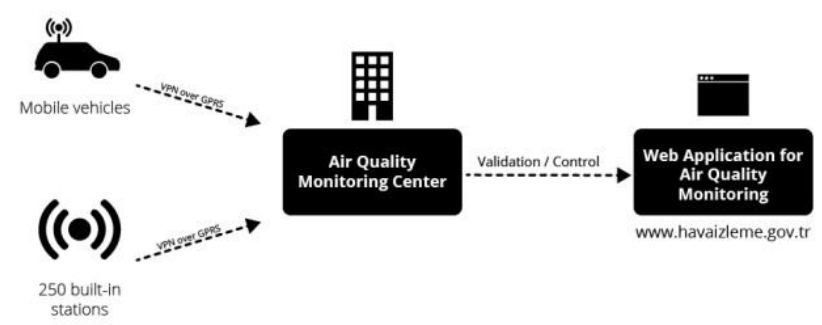

Figure 2. Architecture for TR Air Quality Monitoring System

In this research, National Air Quality Monitoring Web Application was used for obtaining the air quality data. The reporting module of the Web Application is queried manually for $100+$ stations and for every year between 2008-2017. A separate series of queries are used to get geolocation of the monitoring stations. The query results were downloaded using the Web Application in form of CSV files. Following this, the data cleaning is made, information related to the location of the monitoring station is added to each record, and the data is 
formatted in a form that would be easily important by a RDBMS. Following the data acquisition stage the visualisation system is developed by the implementation of the 3-tier system architecture, which is elaborated in the following section.

\subsection{System Architecture}

The web-based visualisation prototype developed in this research consists of 3-tiers (data, business logic and web interface) and 4 different components. The server side of the system consists of a spatial database server (PostGIS), a NodeJS Server for hosting the Cesium 3D Digital Globe environment, and an Apache Server where the business logic is run through server-side PHP scripts (Figure 3).

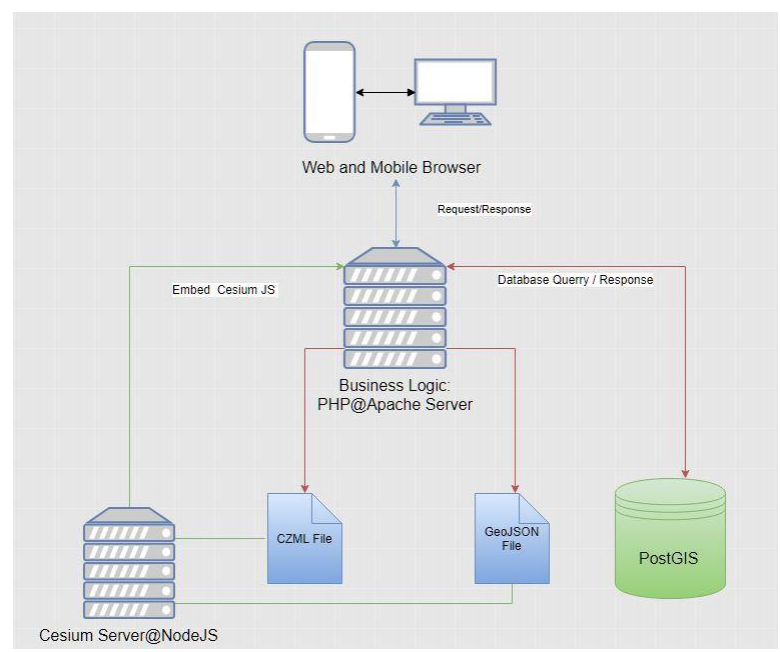

Figure 3: The Prototype System Architecture

The spatial database, NodeJS and Apache Server are running on 3 different Virtual Machines. The system provides a web form interface for user interaction, where a user can send requests regarding their choices on the values that will be visualised and their visualisation approach. The system uses two different visualisation approaches, first one is visualising the pollutant related values through using $3 \mathrm{D}$ primitive solids (i.e. cubes) .The second one is visualising these values using the $2.5 \mathrm{D}$ representations of the buildings that are within a selected circular buffer zone. Regardless of the visualisation approach chosen the system operates as follows. The user provides parameters in relation with the information request (e.g. a request can be for visualising PM10 values of Istanbul Besiktas Monitoring station on 14-Aug-2017 between 17:00 and 18:00 hours), once received, this request is processed by the business logic layer, which forwards a query to the PostGIS database. The database can either return a.)the air quality values only or b.) the air quality values and related building geometries to the Business Logic layer. By using this information and depending on the type of the user's request, the Business Logic layer would generate the 3D geometric representations for visualisation presenting pollutant levels as semantic information. These representations are stored in form of GeoJSON and Cesium CZML files. Once these files are generated at the backend the user is automatically directed to a web page where the resultant files are visualised within a Cesium environment. A mobile or web browser can be used to view and interact with the generated visualisations in Cesium container.

\subsection{Data Storage in a Spatial Database}

The data storage environment of the system is a PostgreSQL Database Server with PostGIS extension. Information regarding the air quality values are stored in a table named "airquality". The number of records in the database is 10.330 .629 . The database contains measurements of air quality between 20082017 that are collected from National Air Quality (AQ) Monitoring Network. The measurements contains hourly readings of the air-quality values. The "airquality" table consists of 11 fields, which store a global id, station name, the date of the measurement, the time of the measurement, PM10 level, CO level, O3 level, NO2 level, a point type geometry representing the station, latitude and longitude of the station. An extract from the table is provided in Figure 4.

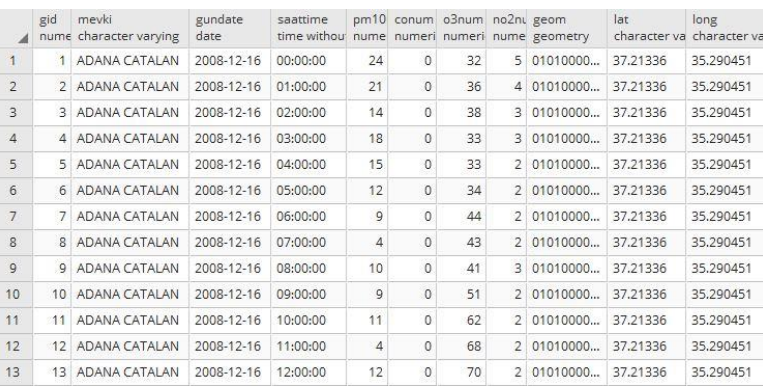

Figure 4: Air Quality Data Table in PostGIS DB

The other table in the PostGIS data store is the "buildings" table, which stores the 2D geometries of the buildings of Istanbul. This table consists of 700.000 records. The fields of the table were the global id, geometry which holds the geometric representation and number of floors attribute which provides the number of floors over the ground level. An extract from the table is provided in Figure 5

\begin{tabular}{|c|r|l|r|}
\hline & $\begin{array}{l}\text { gid } \\
\text { integer }\end{array}$ & $\begin{array}{l}\text { geom } \\
\text { geometry }\end{array}$ & $\begin{array}{l}\text { zeminustuk } \\
\text { numeric (10) }\end{array}$ \\
\hline 1 & 1 & 0106000020 E6100000010.. & 1 \\
\hline 2 & 2 & $0106000020 E 6100000010 \ldots$ & 3 \\
\hline 3 & 3 & $0106000020 E 6100000010 \ldots$ & 6 \\
\hline 4 & 4 & $0106000020 E 6100000010 \ldots$ & 1 \\
\hline 5 & 5 & $0106000020 E 6100000010 \ldots$ & 1 \\
\hline 6 & 6 & $0106000020 E 6100000010 \ldots$ & 0 \\
\hline 7 & 7 & $0106000020 E 6100000010 \ldots$ & 6 \\
\hline 8 & 8 & $0106000020 E 6100000010 \ldots$ & 1 \\
\hline 9 & 9 & $0106000020 E 6100000010 \ldots$ & 3 \\
\hline 10 & 10 & $0106000020 E 6100000010 \ldots$ & 1 \\
\hline 11 & 11 & $0106000020 E 6100000010 \ldots$ & 6 \\
\hline & & & \\
\hline
\end{tabular}

Figure 5: Building Geometries Data Table in PostGIS DB

\subsection{Visualisation of Information}

The visualisation of air quality information is presented following a user query that is sent over a web browser. The visualisation environment is chosen as the Cesium 3D Virtual Globe as it is free to use and as the Cesium API is well documented. The visualisation used a color scheme based on National Air Quality Index documentation. As mentioned earlier, the Turkish National Air Quality Index was adopted from EPA Air Quality Index, and limit values for pollutants are similar to EPA Air Quality Index limits. The Levels of Health Concern and the Coloring Scheme for Turkish Air Quality Index is provided in Table 1. 


\begin{tabular}{|l|l|l|}
\hline $\begin{array}{l}\text { Air Quality Index } \\
\text { (AQI) Values }\end{array}$ & $\begin{array}{l}\text { Levels of } \\
\text { Health Concern }\end{array}$ & Colors \\
\hline $\begin{array}{l}\text { When the AQI is in } \\
\text { this range: }\end{array}$ & $\begin{array}{l}\text { k.air quality } \\
\text { conditions are: }\end{array}$ & $\begin{array}{l}\text {...as bymbolized } \\
\text { by this color: }\end{array}$ \\
\hline 0 to 50 & Good & Green \\
\hline 51 to 100 & Moderate & Yellow \\
\hline 101 to 150 & $\begin{array}{l}\text { Unhealthy for } \\
\text { Sensitive Groups }\end{array}$ & Orange \\
\hline 151 to 200 & Unhealthy & Red \\
\hline 201 to 300 & Very Unhealthy & Purple \\
\hline 301 to 500 & Hazardous & Maroon \\
\hline
\end{tabular}

Table 1: National Air Quality Index Values

In the National Air Quality Index, an index is calculated for five fundamental pollutant types, particle matter (PM10), carbon monoxide $(\mathrm{CO})$, sulfur dioxide (SO2), nitrogen dioxide (NO2) and ozone (O3).The limit values of the index for all these pollutants is provided in Table 2

\begin{tabular}{|c|c|c|c|c|c|c|}
\hline \multirow{2}{*}{ Index } & \multirow{2}{*}{ AQI } & $\mathrm{SO}_{2}\left[\mu \mathrm{g} / \mathrm{m}^{3}\right]$ & $\mathrm{NO}_{2}\left[\mu \mathrm{g} / \mathrm{m}^{3}\right]$ & $\mathrm{CO}\left[\mu \mathrm{g} / \mathrm{m}^{3}\right]$ & $\mathrm{O}_{3}\left[\mu \mathrm{g} / \mathrm{m}^{3}\right]$ & PM10 $\left[\mu \mathrm{g} / \mathrm{m}^{3}\right]$ \\
\hline & & lh.Avg & lh.Avg & Sh.Avg & Sh.Avg & 24h.Avg \\
\hline Good & $0-50$ & $0-100$ & $0-100$ & $0-5500$ & $0-120$ & $0-50$ \\
\hline Moderate & $51-100$ & $101-250$ & $101-200$ & $5501-10000$ & $121-160$ & $51-100$ \\
\hline \begin{tabular}{|c|}
$\begin{array}{c}\text { Unhealthy for } \\
\text { Sensitive } \\
\text { Groups }\end{array}$ \\
\end{tabular} & $101-150$ & $251-500$ & 201-500 & $10001-16000$ & | $161-180$ & $101-260$ \\
\hline Unheatthy & $151-200$ & $501-850$ & 501-1000 & $16001-24000$ & 181-240 & $261-400$ \\
\hline $\begin{array}{l}\text { Very } \\
\text { Unhealthy }\end{array}$ & $201-300$ & $851-1100$ & 1001-2000 & $24001-32000$ & 241-700 & $401-520$ \\
\hline Hazardous & $301-500$ & $>1101$ & $>2001$ & $>32001$ & $>701$ & $>521$ \\
\hline
\end{tabular}

Table 2: National Air Quality Index Limit Values for Different Pollutants

In the system, the air quality information is visualised taking pollutant levels into account. The visualised pollutant levels are coloured according to their "Level of Health Concern" colour. For example, if PM 10 value of 70 would be represented, the colour of the representation would be yellow, or if a PM 10 value of 20 would be represented, the colour of the representation would be green. In addition, in the visualisation, the edges of the geometries are coloured as black or white depending on the time of the (visualised) measurement. The following elaborates on the details of user queries and visualisation of results. The user interface of the system consists of two user forms to generate two different types of queries. The first user form is used in order to visualise the pollutant values in form of primitives/cubes that are placed on top of each other, the second user form is used for visualising these values utilizing the $2.5 \mathrm{D}$ representations of the buildings within a selected circular buffer.

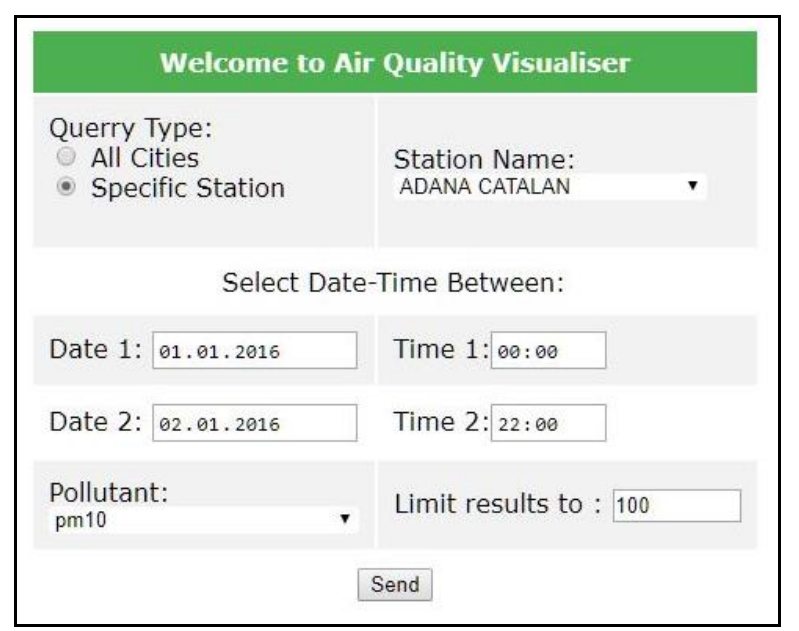

Figure 6: User Input Form

for the visualization of different pollutant levels

In the first user form (Figure 6), the user first selects a query option either to visualise information for all stations/cities or to visualise information for a specific station/city. Following this, the user selects start date/time and end date/time (i.e. a limited time period) and the type of pollutant of which the values would be visualised. Finally, the user is asked to provide a query limit to prevent or reduce session errors caused by response latencies. Once the query is sent the system operates as explained in System Architecture section (of this paper) and produces results in form of GeoJSON and CZML files. The resultant files are visualised in a Cesium environment using Cesium JSAPI as seen in Figures 7 and 8. The figures are showing the results from Adana Catalan and Edirne stations on January 2016 and April 2016. This visualisation shows the information in form of non-overlapping primitives (cubes) that are placed one over another by leaving a constant vertical distance between them to enhance the clarity of the visualisation. The value of the most recent result of the selected time period is represented by the cube on the top and the earliest result is represented by the cube at the bottom. The colour of the faces of the cubes change based on pollutant values, the colour of the edges of the cubes change based on the time of the day. As mentioned, the black/white color of the edges of the cubes represent the time of day (night/day) respectively. Faces of all cubes can appear in green color which indicates the pollutant values are at healthy levels (see. Figure 7 Adana Catalan Station) or can appear in yellow, orange and red colours to illustrate the moderate and unhealthy levels (see. Figure 8 Edirne Station).

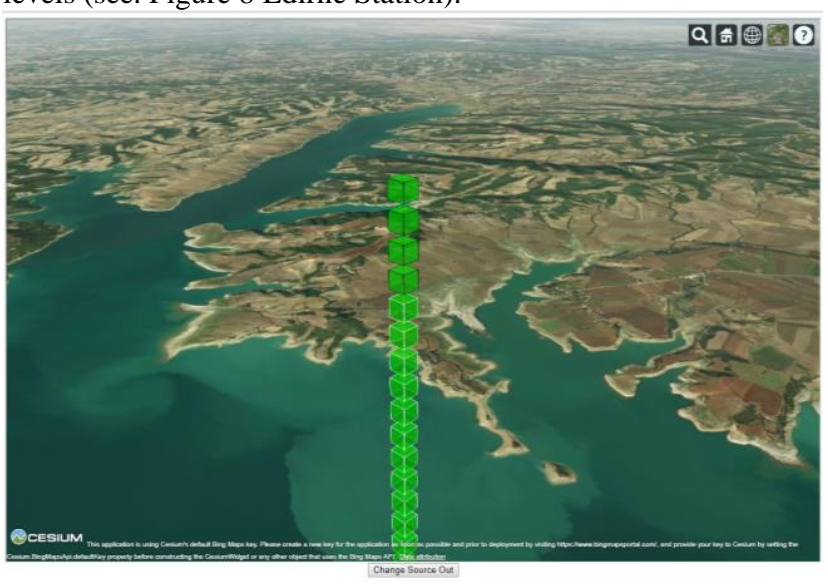

Figure 7: The visualisation of pollutant levels in form of cubes 


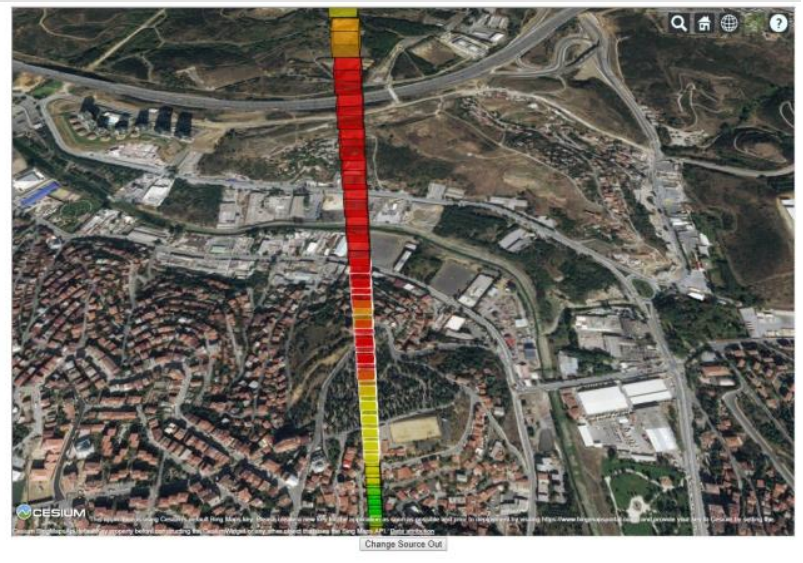

Figure 8: The visualiation of different pollutant levels in form of cubes

The second user form of the application is used for sending queries that will result in visualisation of the pollutant levels using the building geometries that reside in a circular buffer zone, where the station appears in the centre of this circle. In other words, the visualisation highlights values using the geometries of the buildings within a certain distance of a base air quality monitoring station. This visualisation aims to emphasize the impact of pollution on the inhabited areas and people living at the surrounding areas. Figure 9 shows the second user form. In this form, only stations from Istanbul can be selected, as the researchers only can reach and utilize the geometries of the buildings in Istanbul for this research. In addition, as this form is a proof of concept, only PM10 values are visualised for the time being. Once the user selects an air quality monitoring station from the list, the user would select a date and time of a measurement. Following this, the user selects the radius of the circular buffer zone in DMS degrees, and finally in order to prevent session errors and response latencies the user is asked to provide number of results to be returned as a query response limit. Once the query is sent to the server the results are generated using the Business Logic components, saved in form of GeoJSON and CZML files and visualised using the Cesium JSAPI.

\begin{tabular}{|c|c|}
\hline \multicolumn{2}{|c|}{ Welcome to Air Quality Visualiser } \\
\hline Station Name: ISTANBUL BESIKTAS & . \\
\hline Date 1: 01.01 .2016 & Time 1: 14:00 \\
\hline $\begin{array}{l}\text { Limit results to: } \\
110000\end{array}$ & $\begin{array}{l}\text { Radius: } \\
0.01 \mid\end{array}$ \\
\hline \multicolumn{2}{|l|}{ Send } \\
\hline
\end{tabular}

Figure 9: Input Form

for visualization of pollutant levels using 3D building geometries

Two visualisations generated by the system using this second approach is illustrated in Figures 10 and 11. The visualisation is accomplished through extrusion of base geometries of the buildings using the number_of_floors attribute. Similar to the visualisation in the first approach the edges of the buildings are coloured as black/white to express the time of the air quality measurement was made. The polygon color of the buildings indicates the "Level of Health Concern" class of the PM10 value at that certain date/time. For instance, the visualisation in
Figure 10 indicates that there was an unhealthy PM10 value at Istanbul Besiktas station at the chosen time, while at another chosen time as shown in Figure 11 the value indicates that PM10 level is "Unhealthy for Sensitive Groups" for the area shown. For a more detail inspection of the system the readers can find demo videos of the system at https://youtu.be/6ikYACDKDwg, https://youtu.be/JlqvaruikVI, https://youtu.be/a9QVc-X2UN4. The third video illustrates how semantic information can be reached by clicking on the cubes.

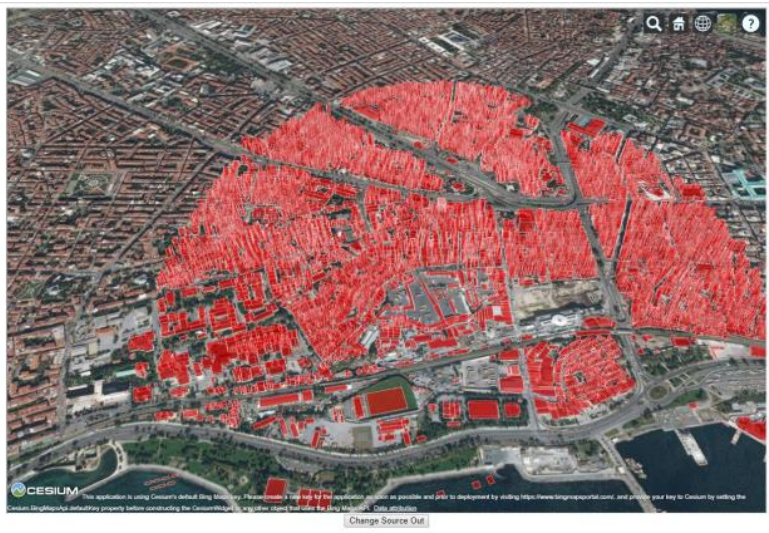

Figure 10: The visualisation of pollutant levels in form building geometries

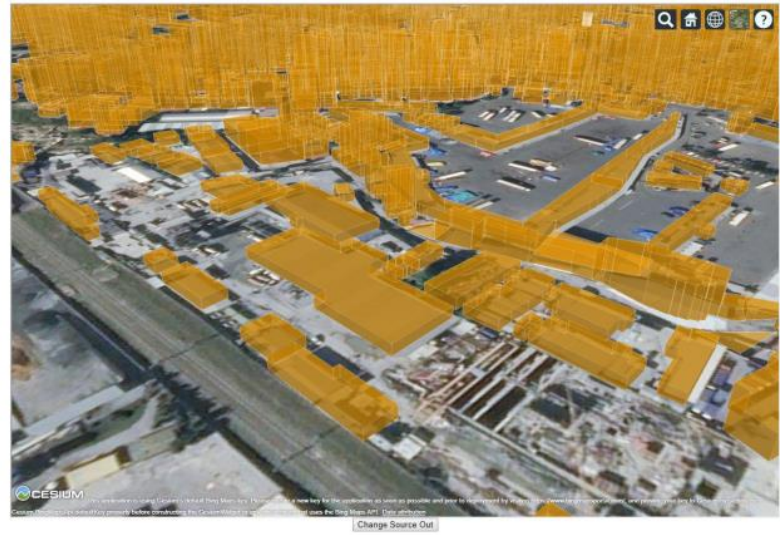

Figure 11: The visualisation of pollutant levels in form building geometries

\section{USABILITY TESTING}

The research aimed to provide a more usable interface/visualisation in order to facilitate the reach to historical air quality information. The potential users of the system is in two groups as i.) general public/home seekers who wants learn about the air quality of a neighbourhood, and ii.) decision makers in urban planning and management. As the system is at the prototype level (not a real-life implementation) the testing of the system did not cover comprehensive techniques such as use-case based evaluation or expert interviews. The testing is done using a simple scale known as SUS. Following the system, the development, the system is tested by utilizing the SUS-System Usability Scale (Brooke, 1996). The SUS is known as a ten-item scale (consisting of 10 questions) giving a global view of usability. The responses can be given using 5-point scale ranging from "strongly agree" to "strongly disagree". In the testing process, the authors explained the system to 10 users who are unexperienced in mapping and GIS (as a sampling from general public). In the second stage, 
demo videos of the system are shown to the users and users can test the live interface of the system. Following this, the users are asked to fill online web form consisting of the 10 questions of the SUS. Usability.gov (2018) mentions that SUS has become an industry standard, with references in over 1300 articles and publications. The average SUS score from all 500 studies is a 68. A SUS score above a 68 would be considered above average and anything below 68 is below average (Measuringu.com, 2011). In the context of this research, the scores of 6 out of 10 testers were above average and 4 out of 10 were below average (see. Figure 12).

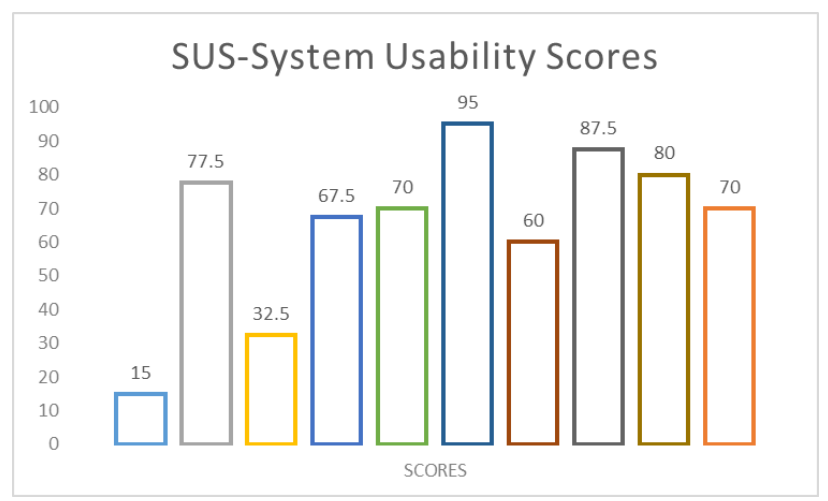

Figure 12. SUSs of the System for each test user

\section{CONCLUSIONS}

As mentioned in the previous research such as Lu et al (2009) the Time-Varying nature of data such as air pollutant level readings, makes the visualisation effort more difficult than real time data. In addition, the amount of data to be queried was huge in the implemented system (i.e. 10.000.000+ records). The key strength of the system is in being a proof-of-concept that spatial high volume data can be visualised through web based tools such as virtual globes. This type of visualisation can facilitate the dissemination of spatial high-volume data through efficient visualisation. For this research, SUS is provided as a quick metric i.e. as an overview on the system quality to the reader. Further studies for measuring the system's efficiency and effectiveness need to be done in a real-life implementation scenario. Multi-tier and distributed system architectures can also be used for this purpose and will provide improvements on system efficiency. Effectiveness of an information system is defined as the extent to which a given information system contributes to achieving organizational goals, i.e. its effect on organizational performance (Hamilton and Chervany, 1981). The model of DeLone and McLean (1992) indicates that the systems quality and the information quality independently and jointly affect the use and user's satisfaction. Additionally, use may affect the degree of user's satisfaction, and vice versa. Use and the user's satisfaction are direct precedents of the individual impact; and finally, this impact on the individual performance should lead to an organizational impact. As this effort is only a prototyping effort, factors such as use and user satisfaction, and individual and organisational impacts can be measured and the effectiveness of the system can be more realistically determined when the system is implemented for a real-life scenario. In addition, sampling from experienced GIS users can also be used in testing the usability of the system. More test subjects would also be needed for the testing of the system that would be developed for a real-life scenario.

The authors acknowledge the fact that the air pollution in not uniformly distributed in space. The visualisations provided in Figure 10 and 11 have a potential to misguide the user as if the pollution is uniformly distributed and the pollutant values would be the same near all buildings that are shown the visualisation, and this can be expressed as the main weakness of the second visualisation approach. In order to achieve more accurate visualisations and impacts of pollution on individuals, the spatial distribution of pollution should be known. Air quality modelling appears as an effective tool for estimating how pollution emitted from a source is distributed depending on atmospheric conditions and topography. In recent years, air quality modelling has become a frequently applied method, especially due to improvements in computer technologies. Inputs of air quality models are emissions inventories, meteorological data and topographic data. The air quality models using this data can estimate the pollutant concentrations depending on the coordinates. The future research will concentrate on visualisation of information that is determined or predicted by different air quality models using city objects in different LODs. Another weakness of the system is at the web forms that are used for sending the queries. The user interface of the system will be enhanced by providing a web map based information seeking tool where the user can select an area from the map and send a query to the system using the coordinates of a bounding box.

\section{REFERENCES}

Ahrens, C., D., 2000. Essentials of Meteorology An Invitation to the Atmosphere, Second Edition., (U.S.A.: Brook/Cole Publishing Company).

Andrienko N., Andrienko G., Gatalsky P., 2003. Exploratory spatio-temporal visualization: an analytical review, Journal of Visual Languages \& Computing, Volume 14, Issue 6, 503-541

Auer M., 2012. Real-Time Web GIS Analysis Using WebGL, International Journal of 3-D Information Modeling, 1(3), 49-61 Boubel, R. W., Fox, D. L., Turner, D. B. ve Stern, A. C. (1994). Fundamentals of Air Pollution (Vol. Third Edition). California: Academic Press.

Brooke, J. 1996 SUS-A quick and dirty usability scale, Usability evaluation in industry, 189 (194), 4-7

Brovelli M., Minghini M., Zamboni G., 2014. Three Dimensional Volunteered Geographic Information: A Prototype of a Social Virtual Globe, International Journal of 3-D Information Modeling, 3(2), 19-34

Christen M., Nebiker S., Loesch B., 2012. Web-Based LargeScale 3D-Geovisualisation Using WebGL: The OpenWebGlobe Project, International Journal of 3-D Information Modeling, $1(3), 16-25$

DeLone, W. H., McLean, E. R. 1992. Information Systems Success: The Quest for the Dependent Variable. Information Systems Research (3), March 1992, pp. 60-95.

Hamilton, S. \& Chervany, N. L. 1981. Evaluating information system effectiveness part I: Comparing evaluation approaches. MIS Quarterly; 5(3), 55-69

Herman,L., Řezník,T., 2015. 3D Web Visualisation of Environmental Information-Integration of Heterogeneous Data Sources When Providing Navigation and Interaction., The International Archives of the Photogrammetry, Remote Sensing and Spatial Information Sciences, Volume XL-3/W3, 2015 
ISPRS Geospatial Week 2015, 28 Sep - 03 Oct 2015, La Grande Motte, France

Gesquière G., Manin A., 2012. 3D Visualization of Urban Data Based on CityGML with WebGL, International Journal of 3-D Information Modeling, 1(3), 1-15

Goetz M. Zipf A., 2012. Towards Defining a Framework for the Automatic Derivation of 3D CityGML Models from Volunteered Geographic Information, International Journal of 3-D Information Modeling, 1(2), 1-16

Homainejad A., 2015. New Approach for Object Detection and Extraction from Digital Images for Providing a 3D Model Applicable in 3D GIS, International Journal of 3-D Information Modeling, 4(3), 34-58

Kaden R., Kolbe T., 2014. Simulation-Based Total Energy Demand Estimation of Buildings using Semantic 3D City Models, International Journal of 3-D Information Modeling, $3(2), 35-53$

Klimke J., Hagedorn B., Döllner J., 2014. Scalable MultiPlatform Distribution of Spatial 3D Contents, International Journal of 3-D Information Modeling, 3(3), 35-49

Miyamura, Hiroko, Suzuki, Yoshio, \& Takemiya (2010). Spatio-temporal visualization. Kashika Joho, 30(suppl1), 269272.

Leeuwen FXR. Update and revision of WHO air quality guidelines for Europe. In: Incecik S, Ekinci E, Yardim F, Bayram A, editors. Proc, 10th Regional IUAPPA Conference on Air Quality Management at Urban, Regional and Global Scales. Zurich, Switzerland: TransTech Publications, 1997. pp. $22-5$.

Li X., Xu W., Zhu Q., Hu J., Hu H., Zhang Y., 2012. A MultiLevel Cache Approach for Realtime Visualization of Massive 3D GIS Data, International Journal of 3-D Information Modeling, 1(3), 37-48

Lu,A., Chen,W., Ribarsky,W., Ebert, D., 2009. Year-Long Time-Varying 3D Air Quality Data Visualization, in Z.W. Ras and W. Ribarsky (Eds.), Advances in Information \& Intelligent Sys., SCI 251, pp. 289-306.

MacNee W., Donaldson K., 1999. Particulate Air Pollution: Injurious and Protective Mechanisms in the Lungs, in Air Pollution and Health, Holgate S.T., Samet J.M., Koren H. S., Maynard R.L. (Ed.) Academic Press, 653-672.

Measuringu.com, 2011. https://measuringu.com/sus/

Jochem R., 2012. Towards Interactive 3D City Models on the Web, International Journal of 3-D Information Modeling, 1(3), 26-36

Ozerbil T., Gokten E., Onder M., Selcuk O., 2015. Oblique Aerial Image Acquisition, 3D City Modeling, 3D City Guide Project for Konya Metropolitan Municipality, International Journal of 3-D Information Modeling, 4(2), 34-47

Polichetti, G., Cocco, S., Spinalli, A., Trimarco, V., Nunziata, A., 2009. Effects of particulate matter (PM10, PM 2.5 and PM1) on the cardiovascular system, Toxicology, 261, 1-8.
San José R., Pérez J.L., González R.M., 2012. Advances in 3D visualization of air quality data, Usage, Usability, and Utility of 3D City Models, 2012-10-24

Spellman, F.R., 2009. The Science of Air Concepts and Applications, Second Edition., (U.S.A.: CRC Press).

Tsiliakou E., Labropoulos T., Dimopoulou E., 2014. Procedural Modeling in 3D GIS Environment, Procedural Modeling in 3D GIS Environment International Journal of 3-D Information Modeling, 3(3), 17-34

Usability.gov, 2018. https://www.usability.gov/how-to-andtools/methods/system-usability-scale.html

Zardiny A.,Hakimpour F., 2015. 3D Web Services for Visualization and Data Sharing in 3D Cadastre, International Journal of 3-D Information Modeling, 4(4), 1-15

Zhu W., Wursthorn S., Simons W., Nichersu A., 2016. Integration of CityGML and Air Quality Spatio-Temporal Data Series via OGC SOS, Geospatial Sensor Webs Conference 2016, 12-16 